\section{Das periphere olfaktorische System von Vertebraten: Molekulare, strukturelle und funktionelle Grundlagen des Riechens}

\author{
Ivan Manzini und Sigrun Korsching
}

\begin{abstract}
Zusammenfassung
Der Geruchssinn liefert Menschen und Tieren eine Vielzahl von Informationen über die Umgebung, er trägt zur Orientierung und Warnung bei, steuert die Nahrungsaufnahme, die Wahl des Sexualpartners und beeinflusst maßgeblich das innerartliche Sozialverhalten. Die Wahrnehmung von Geruchsstoffen beginnt mit der Bindung der Geruchsmoleküle an spezialisierte Rezeptormoleküle der Plasmamembran, die fast ausnahmslos zu der Superfamilie der G-Protein-gekoppelten Rezeptoren (GPCR) gehören. Insgesamt sind bisher fünf verschiedene Familien von Geruchsrezeptoren identifiziert worden, darunter die mit Abstand größte Genfamilie überhaupt mit über tausend Genen in Nagern. Für diese Familie ist die Signaltransduktionskaskade mittlerweile gut charakterisiert. Drei Arten von Rezeptorneuronen, die ziliierten, mikrovillären und Kryptneurone lassen sich anatomisch und molekular voneinander unterscheiden. Rezeptorneurone beschränken sich in der Regel auf die Expression eines einzigen Geruchsrezeptorgens, und zudem senden Neurone mit übereinstimmend ausgewähltem Rezeptor ihre Axone in eine gemeinsame Zielstruktur, einen Glomerulus, wodurch in der ersten olfaktorischen Gehirnregion, dem Bulbus olfactorius, eine rezeptotope Karte entsteht. Dieser Review gibt zum einen eine allgemeine Übersicht der peripheren Detektion von Geruchsstoffen und fokussiert zum anderen auf die in den letzten Jahren neu gewonnenen Erkenntnisse, unter anderem zur peripheren Modulierung olfaktorischer Signale.
\end{abstract}

\begin{abstract}
The peripheral olfactory system of vertebrates: molecular, structural and functional basics of the sense of smell

The sense of smell provides people and animals with a multitude of informations about their environment, which help them in navigation, warn of potential threats, control food intake, the choice of sexual partners and significantly influence the intraspecies social behavior. The perception of odors begins with the binding of odor molecules to specialized olfactory receptor proteins, which nearly all belong to the superfamily of $\mathbf{G}$ protein-coupled receptors. Altogether five different olfactory receptor gene families have been described so far, among them the largest gene family in the genome with over one thousand genes in rodents. For this family the signal transduction cascade coupled to the receptors has already been well characterized. Three different classes of receptor neurons - ciliated, microvillous and crypt receptor neurons - can be distinguished by anatomical and molecular characteristics. Generally an individual receptor neuron expresses only a single olfactory receptor gene, and olfactory receptor neurons that express the same receptor converge into a common target structure, a glomerulus, which generates a receptotopic map in the first olfactory brain region, the olfactory bulb. This review on the one hand gives a general overview over the peripheral detection of odorants and on the other hand focuses on recent advances in the field, including new findings regarding the peripheral modulation of olfactory signals.
\end{abstract}

Keywords: olfactory receptor neurons; odorant receptor; signal transduction; peripheral modulation; olfactory wiring; behaviour

\section{Vorbemerkung}

Der Geruchssinn ist phylogenetisch alt, seine Ursprünge liegen in der Detektion von Umgebungsmolekülen und reichen bis zur Chemotaxis der Bakterien zurück. Im engeren Sinne wird als Geruchssinn ein spezialisierter Sinn u.a. der Vertebraten und Arthropoden bezeichnet, der sich durch molekulare und anatomische Eigenständigkeit von dem zweiten spezialisierten chemosensorischen Sinn, dem Geschmackssinn unterscheiden lässt. In erster Näherung unterscheidet sich der Geruchssinn durch höhere Sensitivität und analytische statt kategorisierende Signalverarbeitung vom Geschmackssinn. Dieser Review behandelt die initialen Vorgänge bei der Geruchsstoffwahrnehmung von Wirbeltieren bis hin zur Repräsentation von Geruchsstoffen im Riechkolben, dem Bulbus olfactorius.

\section{Struktur und Evolution der Geruchsrezeptorgene}

Während der Evolution der Wirbeltiere entstanden nacheinander verschiedene Geruchsrezeptorfamilien, bzw. wurden Mitglieder verschiedener Familien als Geruchsrezeptorgene rekrutiert, was jeweils zu einer mehr oder minder starken Expansion der betreffenden Familie führte. Meist sind Genfamilien von Geruchsrezeptoren durch dynamische Evolution und dementsprechend ausgeprägte Speziesspezifität der Rezeptor-Repertoires gekennzeichnet. Es gibt sogar Hinweise auf positive Selektion, also Selektion auf Diversität der Rezeptorgene. Gegenwärtig sind für Wirbeltiere fünf solcher Rezeptorgenfamilien bekannt (Abbildung 1), die in vielen Spezies durch bioinformatische Methoden auf der Grundlage der Genomsequenz vorhergesagt werden konnten (Mombaerts 2004).

$\mathrm{Zu}$ den Geruchsrezeptorgenen zählen die zuerst entdeckte und daher mit dem generischen Namen versehene Familie der Geruchsrezeptoren ('odorant receptors', ORs), zwei Familien von vomeronasalen Rezeptoren, die nach dem Expressionsort in Säugern benannt wurden (V1R und V2R, für die Fischhomologen findet man auch die Bezeichnung ORA bzw. OlfC), eine Familie von aminergen Rezeptoren ('trace amine-associated receptors', TAAR) sowie die erst 2009 als Geruchsrezeptoren identifizierten FormylpeptidRezeptoren (FPR), s. (Mombaerts 2004; Tirindelli et al. 2009; Rivière et al. 2009). Die vomeronasalen Rezeptoren werden manchmal als Pheromonrezeptoren von den eigentlichen Geruchsrezeptoren unter- 
schieden, was mittlerweile als eher artifizielle Unterteilung erscheint und im Weiteren hier nicht berücksichtigt wird. Pheromone sind organische Moleküle, die der biochemischen Kommunikation innerhalb einer Spezies dienen (Botenstoffe zwischen verschiedenen Spezies werden als Kairomone bezeichnet), der Unterschied zu 'normalen' Geruchsstoffen liegt also nicht notwendigerweise in ihrer chemischen Natur oder ihren Rezeptoren, sondern viele Ebenen später in der Ankopplung bestimmter Verhaltensweisen. Bis auf die dem metabotropen Glutamatrezeptor ähnlichen V2R-Gene aus der Gruppe C der GPCR gehören alle anderen Familien zu den rhodopsinähnlichen GPCR (Gruppe A), bzw. sind ihnen ähnlich (V1RFamilie). Die meisten Geruchsrezeptoren sind monoexonisch, mit Ausnahme mancher Repräsentanten der V1R/ORA-Familie aus Fischen und den V2R-Genen, die einen für diese Familie und eng verwandte Gene charakteristischen Aufbau aus sechs Exonen aufweisen.

In verschiedenen Spezies kann der Umfang einer Familie zwischen Null und mehreren hundert Mitgliedern variieren (Abbildung 1). Den Rekordhalter stellt die Familie der ORs dar, mit über tausend Genen in Nagern (Mombaerts 2004). Es wird vermutet, dass die Familiengröße mit der Bedeutung des Geruchssinns für die jeweilige Spezies korreliert, jedoch fehlen bisher belastbare Daten dazu, u.a. weil die Deorphanisierung der Rezeptoren sich als schwierig erwiesen hat. Die Familiengrößen wurden zum einen durch drei Genomverdoppelungen in der Vertebratenentwicklung beeinflusst, wovon die dritte nur Knochenfische betrifft. Wichtiger sind allerdings die häufigen Genverluste und lokalen Genduplikationen, wobei letztere in der oft beobachteten Anordnung der Geruchsrezeptorgene im Genom in großen Clustern resultieren. Beides zusammen kann dazu führen, dass in verwandten Spezies sehr unterschiedliche Familiengrößen vorliegen, z.B. gibt es im Menschen kein einziges intaktes V2R-Gen und nur wenige Pseudogene, während in der Maus knapp hundert intakte Gene und noch einmal doppelt so viele Pseudogene vorliegen (Mombaerts 2004). Die Häufigkeit von Pseudogenen ist oft sehr groß, vermutlich weil der Ausfall einzelner Rezeptoren meist keinen Selektionsnachteil darstellt. Allerdings sollte erwähnt werden, dass die konkreten Zahlen sehr stark von der Qualität der verwendeten Datenbanken und Vorhersagealgorithmen abhängen.

Die V1R-verwandten ORA-Gene der Fische zeigen ein drastisch abweichendes Verhalten: Es handelt sich um eine klei-

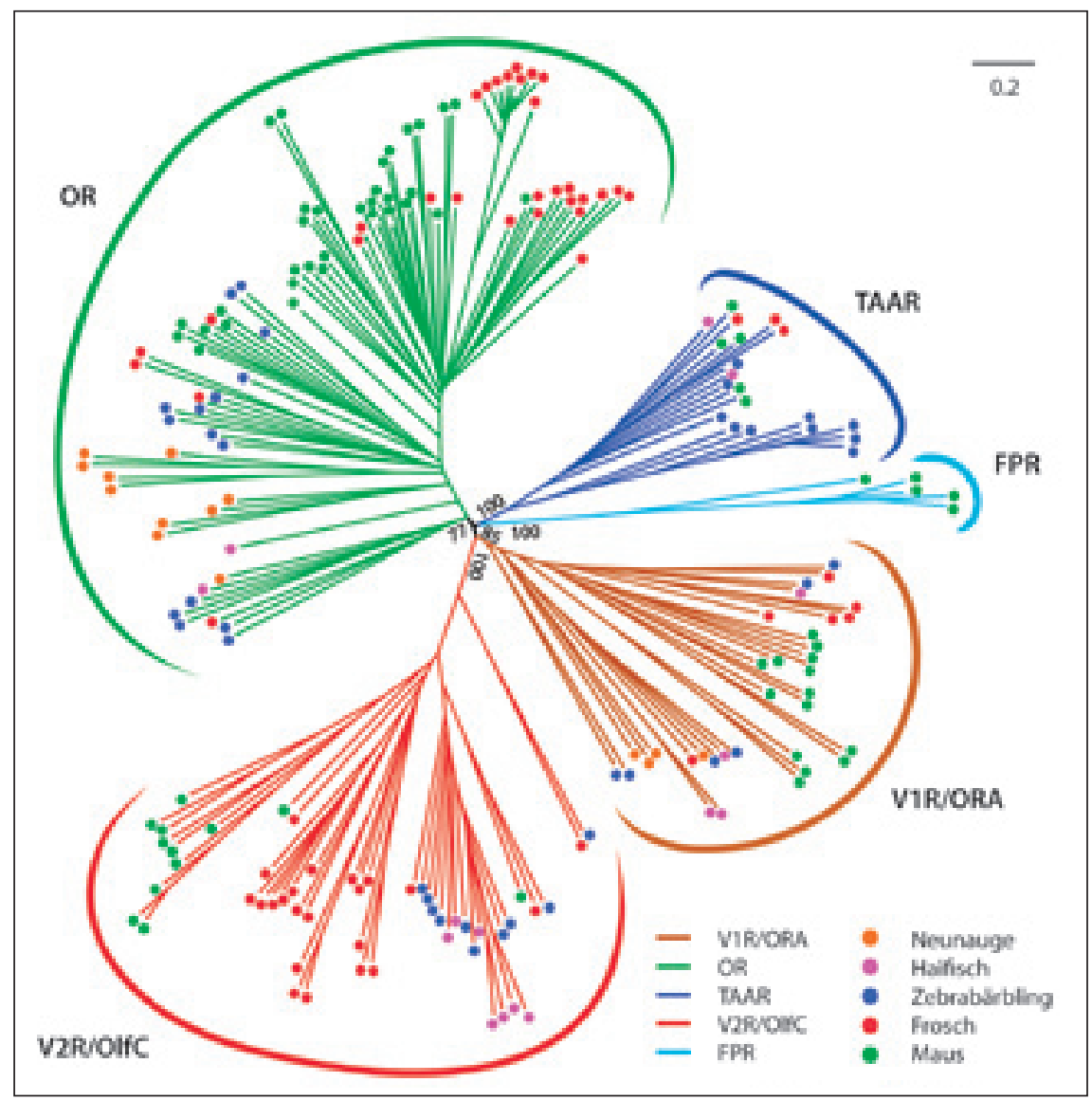

Abb. 1: Evolutionäre Verwandschaft der Geruchsrezeptorgene

Phylogenetischer Baum, der die Verwandschaft der fünf GPCR-Rezeptorgenfamilien zeigt. OR, ,odorant receptor' Protein; TAAR, ,trace amine“ assoziierte Rezeptoren; V1R und V2R, vomeronasale Rezeptoren Typ 1 und 2; ORA, V1R-homologe Fischrezeptoren; OIfC, V2R-homologe Fischrezeptoren; FPR, Formylpeptidrezeptoren. Der Übersichtlichkeit halber wurde für Genfamilien größer als 20 Gene eine zufällige Auswahl von Rezeptoren verwendet. Die Spezies sind farbkodiert; blau, Zebrabärbling, ein Teleosteer; rot, Krallenfrosch, ein Amphibium; grün, Maus, ein Säuger; magenta, Elefantenhai, ein Knorpelfisch; braun, Neunauge, ein kieferloser Fisch. Die Proteinsequenzen wurden mit MAFFT ausgerichtet, und der Baum mittels NJ-Algorithmus unter Verwendung von 1000 Bootstrap-Zyklen berechnet.

ne Familie aus sechs hochkonservierten Genen, die möglicherweise schon vor der Entstehung der Knochenfische in derselben Größe bestanden hat, und auf die also weder die letzte Genomverdoppelung noch lokale Genduplikation einen Einfluss hatten.

Die fünf Rezeptorgenfamilien sind zu unterschiedlichen Zeitpunkten während der Evolution der Vertebraten entstanden bzw. vom olfaktorischen System kooptiert worden. Die phylogenetisch ältesten Familien sind die OR- und V1R-Familien, die schon vor der Abspaltung der kiefertragenden Fische von den kieferlosen (z.B. Neunauge) mit mehreren Vertretern nachweisbar sind, während die ersten Vertreter der TAAR- und V2R-Rezeptoren erst in den Knorpelfischen (z.B. Haifisch) gefunden wurden (Abbildung 1). Interessanterweise sind allerdings den TAAR-Genen verwandte aminerge Rezeptorgene bereits im Neunauge als olfaktorische Rezeptorgene rekrutiert worden, vermutlich unabhängig von den TAAR-Genen. Der evolutionär jüngste $\mathrm{Zu}-$ wachs sind die FPR-Gene, die in Säugern als immunologisch bedeutsame Rezeptorgruppe entstanden sind, aber nur in Nagern als Geruchsrezeptorgene nachgewiesen wurden (Rivière et al. 2009).

Schließlich sollte noch erwähnt werden, dass neben den fünf hier behandelten GPCR-Familien auch zwei membrangebundene Guanylatzyklasen als Geruchsrezeptorgene in Säugern diskutiert werden (Tirindelli et al. 2009), bei denen es sich um eine phylogenetisch deutlich ältere Genfamilie handelt, die bereits in Invertebraten chemosensorische Funktionen hat. 


\section{Expressionsmuster der Rezeptorgene} und zugrunde liegende Mechanismen

Die bisherigen Untersuchungen haben ganz überwiegend gezeigt, dass jedes olfaktorische Rezeptorneuron ein einziges Rezeptorgen aus einer der fünf Familien exprimiert (monogene Expression). Benachbarte Rezeptorneuronen exprimieren im Allgemeinen unterschiedliche Gene, was das charakteristische gesprenkelte Expressionsmuster zur Folge hat. Damit bestimmt die Zahl der verschiedenen Rezeptorgene die Anzahl der Subpopulationen von Rezeptorneuronen und folglich die Zahl der Eingangskanäle in den Bulbus olfactorius, die erste Gehirnregion, die mit der Verarbeitung der geruchlichen Information befasst ist. Für Mäuse sind das allein in der OR-Familie ca. 1000 individuelle Rezeptorgene, zu denen noch mehrere hundert Gene aus den anderen vier Familien dazukommen (Mombaerts 2004).

Die monogene Expression, auch als EinNeuron-Ein-Rezeptor-Regel bekannt, stellt ein interessantes regulatorisches Problem dar, da jedes einzelne Neuron ja eine Auswahl des zu exprimierenden Rezeptors aus einer großen Anzahl gleichartiger Rezeptorgene treffen muss. Die beteiligten Mechanismen sind komplex, mehrere Beobachtungen legen nahe, dass es sich bei der Rezeptorauswahl um einen mehrstufigen Prozess handelt. In verschiedenen Typen von Rezeptorneuronen (s.u.) werden unterschiedliche Familien olfaktorischer Rezeptorgene exprimiert, sodass es familienspezifische regulatorische Elemente geben sollte (Ausnahmen gibt es allerdings in jeder Familie). Zusätzlich ist die räumliche Verteilung der Expression einzelner Rezeptorgene innerhalb einer Familie unterschiedlich. Innerhalb der Riechschleimhaut werden einzelne OR Rezeptorgene in sogenannten Expressionszonen oder-Domänen exprimiert, die teils relativ deutlich voneinander abgegrenzt sind (die Klasse 1 und Klasse 2 Geruchsrezeptoren der Säuger), teilweise aber auch massiv überlappen, insbesondere, aber nicht begrenzt auf niedere Vertebraten. Schließlich weist die 'gesprenkelte' Verteilung der einen bestimmten Rezeptor exprimierenden Neuronen auf ein stochastisches Element in der Regulierung hin.

Bisher wurde davon ausgegangen, dass negative Rückkopplung durch den ausgewählten Rezeptor die Expression anderer Rezeptoren verhindern kann (Mori und Sakano 2011). Zusätzlich wurde die Existenz langreichweitiger Enhancerregionen (H region) nachgewiesen, die zur exklusiven Expression eines einzigen Gens jedenfalls aus der im Einzugsbereich dieses Enhancers liegenden Gruppe von Genen führen könnten. Kürzlich konnte jedoch gezeigt werden, dass olfaktorische Rezeptorgene in den Rezeptorneuronen (und nur dort) allgemein reprimiert sind durch eine bestimmte Histonmethylierung (Magklara et al. 2011), sodass man auch vermuten könnte, dass die beobachtete monogene Expression dadurch zustandekommt, dass das Überwinden der allgemeinen Repression ein sehr unwahrscheinlicher, also seltener Vorgang ist. In diesem Fall würde man eine gelegentliche Ko-Expression erwarten, die aber unter einer Frequenz von 0.001 liegen sollte. Monogene Expression wurde auch im Zebrabärbling nachgewiesen, wenngleich für eine OR-Unterfamilie die gleichzeitige Expression von nahe verwandten Genen darauf hindeuten könnte, dass die Expression in niederen Vertebraten weniger restriktiv geregelt ist.

Geruchsrezeptoren der Säuger werden zudem monoallelisch exprimiert, hier ist eine gleichzeitige Expression beider parentaler Allele nur in ungefähr ein Promille aller untersuchten Zellen gefunden worden. Da unterschiedliche Allele sich durchaus in ihrem Ligandenbindungsspektrum unterscheiden können, dient die monoallelische Expression also ebenfalls dem Zweck einer Begrenzung der Reaktivität der Rezeptorneuronen. Monoallelische Expression ist entgegen früherer Vorstellungen weitverbreitet im Genom, der Mechanismus könnte, muss aber nicht unabhängig sein von dem der monogenen Expression (Mombaerts 2004; Mori und Sakano 2011; Magklara et al. 2011).

Abweichend von den hier beschriebenen Expressionsmustern findet man eine paarweise Ko-Expression von Mitgliedern einer bestimmten V2R-Unterfamilie mit jeweils einem Mitglied aus den anderen Unterfamilien. Eine breite Expression der homologen Gene im Zebrabärbling (OlfCa1, OlfCc1) lässt ebenfalls auf eine Ko-Expression mit den spezifisch exprimierten OlfC-Genen schließen. Die Funktion ist nicht bekannt, könnte jedoch ähnlich der des ebenfalls breit exprimierten OR83b der Insekten sein (s. Artikel von Sachse und Krieger, diese Ausgabe).

\section{Ligandenbindungseigenschaften der Geruchsrezeptoren}

Die Bindungseigenschaften der Rezeptoren legen den virtuellen Raum aller möglichen Geruchsstoffe fest. Prinzipiell lässt sich jeder Geruchsstoff und jede Mischung von Geruchsstoffen als ein Punkt in einem virtuellen Geruchsraum auffassen, dessen Achsen durch die vorhandenen Geruchsrezeptoren aufgespannt werden. In der Praxis ist eine solche Darstellung nicht möglich, da bisher nur wenige Geruchsrezeptoren deorphanisiert werden konnten. Zum einen liegt das sicher an der Vielfalt möglicher Liganden, aber technische Gründe spielen auch eine große Rolle, insbesondere die meist ineffektive Expression von olfaktorischen Rezeptoren in heterologen Systemen aufgrund von intrazellulären Transportproblemen. Hier konnten, u.a. durch Ko-Expression von Transportermolekülen und Einfügung von effizienten Signalsequenzen die Expressionsraten von olfaktorischen Rezeptoren in heterologen Systemen stetig verbessert werden (s. Mombaerts 2004; Saito et al. 2009).

Die bisherigen Ergebnisse zeigen, dass die Mehrzahl der deorphanisierten Rezeptoren eine deutliche Spezifität für einige wenige Liganden aufweisen, die oft, aber nicht immer, chemisch miteinander verwandt sind. Umgekehrt werden einzelne Liganden überwiegend durch mehr als einen Rezeptor erkannt, wobei die Affinität um mehrere Größenordnungen variieren kann (Mombaerts 2004; Saito et al. 2009). Hierdurch ergibt sich eine kombinatorische Kodierung, in der bestimmte Geruchsstoffe jeweils durch unterschiedliche Kombinationen von aktivierten Rezeptoren repräsentiert werden.

Davon abweichend sind manche Rezeptoren, insbesondere Pheromonrezeptoren hochspezifisch und erkennen nur einen einzigen Geruchsstoff aus der getesteten Palette (s. Haga et al. 2010), während andere Rezeptoren durch ein ungewöhnlich breites Ligandenspektrum hervorstechen, so z.B. SR1, der Hauptrezeptor aus dem Septalorgan. Das Verständnis der Liganden-RezeptorWechselwirkung steht erst in den Anfängen, da die Strukturanalyse sich bisher auf Modellierung und Mutationsanalyse beschränken muss, die zudem bisher nur an einer Handvoll von Geruchsrezeptoren durchgeführt wurden. Vermutlich am besten verstanden ist der I7-Rezeptor, dessen Ortholog in der Ratte durch einen aliphatischen Aldehyd mittlerer Kettenlänge (n-Oktanal) optimal aktiviert wird. Verschiedentlich sind kompetitive Antagonisten nachgewiesen worden, also Stoffe, die die Bindung anderer Geruchsstoffe blockieren können, ohne selber aktivierend zu wirken. Der Status als Antagonist hängt von dem anzukoppelnden G-Protein ab, was einen ersten Hinweis auf die weiteren Vorgänge nach der Bindung des Geruchsstoffes darstellt, wenngleich in keinen Fall geklärt ist, welche intramolekularen Vorgänge nach der Bindung eines Liganden zur Aktivierung des Rezeptors führen.

Die Bandbreite der Geruchsstoffe ist sehr groß und reicht von Gasen $\left(\mathrm{H}_{2} \mathrm{~S} ; \mathrm{CO}_{2}\right.$, wahrgenommen als Hydrogencarbonat, Rezeptoren GC-D und GC-G) über vielfältige niedermolekulare organische Stoffe, teils mit Pheromoncharakter (u.a. Aldehyde, Amine, Aminosäuren, Alkohole, Gallensäuren, Nukleotide) bis hin zu Peptiden (u.a. MHC-Peptide, 
Rezeptoren der V2R-Familie), wobei die nichtflüchtigen Verbindungen entweder über direkten Kontakt aufgenommen werden oder nur bei aquatischen Spezies als Geruchsstoffe fungieren.

\section{Olfaktorische Rezeptorneurone}

Olfaktorische Rezeptorneurone in Vertebraten sind bipolare Neurone mit einem Axon, das in den Bulbus olfactorius projiziert, und einem Dendriten, der in einem sogenannten Riechköpfchen endet, welches der Geruchswahrnehmung dienende spezialisierte Strukturen trägt, wobei es sich um nichtmotile Zilien oder Mikrovilli handelt.

In Säugern sind ziliierte und mikrovilläre Rezeptorneuronen in verschiedenen olfaktorischen Organen lokalisiert, die ziliierten Zellen in der Hauptriechschleimhaut und dem nach seiner Lage auf dem Septum benannten Septalorgan, ziliierte Zellen abweichender Morphologie im extrem anterior gelegenen Grüneberg Ganglion, und zwei anatomisch und funktional unterschiedliche Populationen mikrovillärer Neuronen in der zweischichtigen vomeronasalen Riechschleimhaut (apikale und basale Schicht). Demgegenüber besitzen Fische keine separaten olfaktorischen Organe, sondern eine einzige Riechschleimhaut, in der mikrovilläre und ziliierte Neuronen vereint sind. Bei Amphibien beginnt die räumliche Trennung beider Zelltypen, es existiert ein vomeronasales Organ mit mikrovillären Rezeptorneuronen, jedoch sind in der Hauptriechschleimhaut in vielen Spezies neben den ziliierten auch noch mikrovilläre Neuronen vorhanden.

Ein dritter Typus von Rezeptorneuronen, die sogenannten Kryptzellen, zeichnet sich aus durch seine globuläre Morphologie, die Abwesenheit eines erkennbaren Dendriten sowie die gleichzeitige Anwesenheit von Zilien und Mikrovilli und ist bisher nur bei Fischen gefunden worden, wenngleich zumindest oberflächliche morphologische Ähnlichkeit mit den ziliierten Neuronen des Grüneberg-Ganglions besteht.

Mit einigen Ausnahmen werden die fünf olfaktorischen Rezeptorgenfamilien der Säuger jeweils spezifisch in bestimmten Rezeptorneuron-Subpopulationen exprimiert. Die ORFamilie wird ganz überwiegend in ziliierten Rezeptorneuronen der Hauptriechschleimhaut exprimiert, ebenso die TAAR-Familie. Abweichend hiervon werden wenige ORs auch im Septalorgan, im Grüneberg-Ganglion und im vomeronasalen Organ exprimiert, sowie manche TAARs ebenfalls im Grüneberg-Ganglion. V1R-, V2R- und FPR-Gene werden in mikrovillären Rezeptorneuronen exprimiert, ein V2R-Rezeptor auch im Grüneberg-Ganglion. Für die OR- und V2R-homologen OlfC-
Gene der Fische gelten die entsprechenden Zuordnungen zu ziliierten bzw. mikrovillären Rezeptorneuronen.

Aufgrund der monogenen Expression der olfaktorischen Rezeptorgene sollten die Antworteigenschaften der Rezeptorneuronen in erster Näherung denen der zugrunde liegenden Rezeptormoleküle ähneln. In der Tat findet man auch auf der Ebene der Rezeptorneuronen überwiegend die kombinatorische Repräsentation von Geruchsstoffen durch Aktivierung einer Untergruppe von Rezeptorneuronen, wobei einzelne Rezeptorneuronen auch hier wieder sehr unterschiedliche Affinitäten für denselben Geruchsstoff aufweisen können (Nara et al. 2011). Gelegentlich werden auch hochspezifische Rezeptorneuronen beobachtet, meist für Stoffe, die als Pheromone oder Kairomone gelten (Nara et al. 2011).

\section{cAMP-vermittelte Signaltransduktion in olfaktorischen Rezeptorneuronen der Säuger}

Die an die olfaktorischen Rezeptoren des OR-Typs gekoppelte Transduktionskaskade ist in den ziliierten Rezeptorneuronen der
Säuger am besten erforscht (Abbildung 2). Die Bindung eines Geruchsstoffmoleküls an einen Rezeptor führt zur Aktivierung eines spezifischen olfaktorischen G $\alpha$ Proteins $\left(\mathrm{G}_{\mathrm{olf}}\right)$, welches seinerseits eine membranständige Adenylatcyclase des Typs III aktiviert, die wiederum den Botenstoff cAMP synthetisiert, der an nichtselektive CNG-Kanäle (CNG, für 'cyclic nucleotidegated') bindet und sie dadurch öffnet. Durch diese Kanäle strömen in der Mukusschicht der Riechschleimhaut vorhandene $\mathrm{Na}^{+}$- und $\mathrm{Ca}^{2+}$-Ionen in das Zilienlumen ein, was zu einer Depolarisiation der Zilienmembran und somit zu einem Rezeptorpotenzial führt. Die einströmenden $\mathrm{Ca}^{2+}$-Ionen öffnen daraufhin kalziumaktivierte Chloridkanäle, durch die $\mathrm{Cl}^{-}$-Ionen das Zilienlumen Richtung Mukusschicht verlassen. Die molekulare Identifizierung war langwierig, nachdem zwischenzeitlich ein Mitglied der Bestrophinfamilie als Kandidat galt, konnte schließlich Ano2/TMEM16b als Chloridkanal der ziliierten (und mikrovillären) Rezeptorneuronen identifiziert werden. Bisher wurde vermutet, dass diese Chloridkanäle eine bis zu zehnfache Verstärkung des Signals

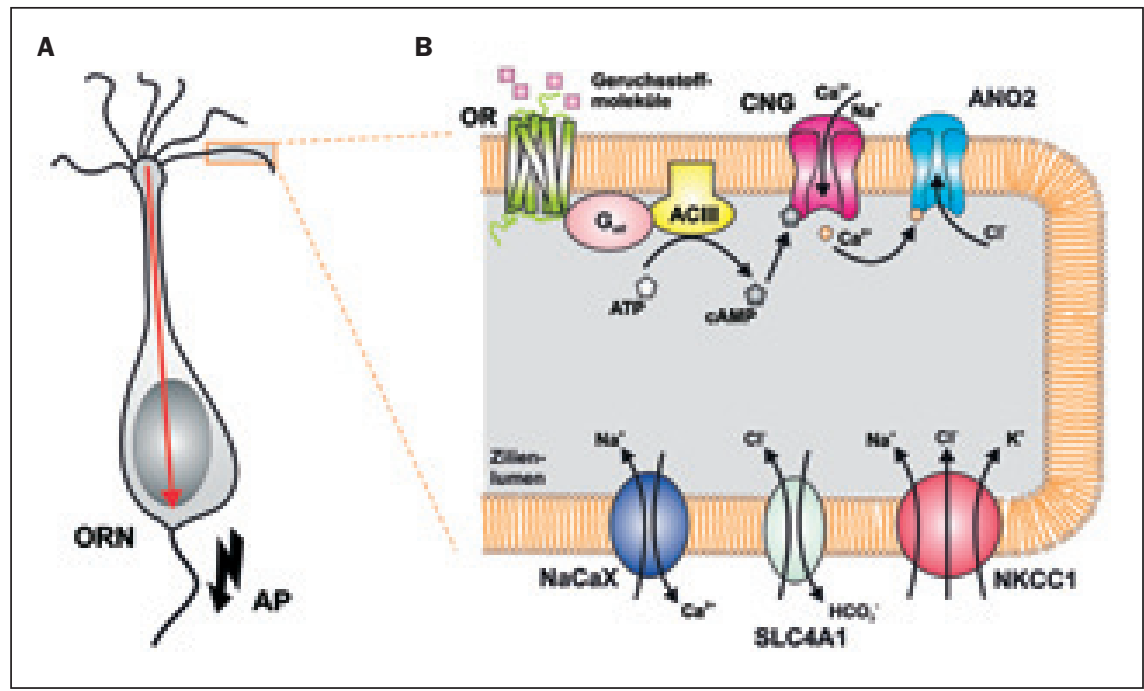

Abb. 2: Signaltransduktionskaskade der olfaktorischen Rezeptoren (OR)

A) Schematische Darstellung eines olfaktorischen Rezeptorneurons (ORN). Olfaktorische Rezeptorneurone sind bipolare Neurone, deren Dendrit an der Oberfläche der Riechschleimhaut ein Riechköpfchen mit sensorischen Zilien besitzt. In den Zilien findet die olfaktorische Transduktion statt (siehe B). Das durch die Transduktion entstandene Rezeptorpotenzial wird elektrotonisch (roter Pfeil) zum Axonhügel weitergeleitet, wo nach Erreichen der Schwelle von spannungsabhängigen $\mathrm{Na}^{+}$-Kanälen eine Salve von Aktionspotenzialen (AP) ausgelöst wird. B) Schematische Darstellung eines olfaktorischen Ziliums. Die Darstellung illustriert, welche Prozesse ablaufen, wenn ein Geruchsstoffmolekül an einen olfaktorischen Rezeptor bindet und wie die lonenkonzentrationen im Zilium aufrecht erhalten werden. Eine genaue Beschreibung dieser Prozesse kann im Text gefunden werden. Verwendete Abkürzungen: ANO2, kalziumaktivierter Chloridkanal; ACIII, Adenylatcyclase des Typs 3; ATP, Adenosintriphosphat; cAMP, zyklisches Adenosinmonophosphat; CNG, zyklisch-nukleotid-gesteuerter lonenkanal; Golf, olfaktorisches G-Protein; $\mathrm{NaCaX}, \mathrm{Na}^{+} / \mathrm{Ca}^{2+}$ Austauscher; NKCC1, $\mathrm{Na}^{+} / \mathrm{K}^{+} / 2 \mathrm{Cl}-\mathrm{Cotransporter}$; SLC4A1, $\mathrm{Cl}-/ \mathrm{HCO}_{3}-\mathrm{Austauscher}^{-}$ 
bewirken, was jetzt angezweifelt wird, da ein Knockout dieses Kanals die Chloridströme vollständig eliminieren konnte, jedoch nicht zu einer wesentlichen Verschlechterung der Signaltransduktion führte (Billig et al. 2011).

Verschiedene Transporter dienen zur Rückführung der elektrochemischen Gradienten in den Ruhezustand, sowohl was Chloridionen, als auch mono- und divalente Kationen betrifft. Zum einen werden in der Zilienmembran ein $\mathrm{Na}^{+} / \mathrm{K}^{+} / 2 \mathrm{Cl}^{-}$Cotransporter (NKCC1) und ein $\mathrm{Cl}^{-} / \mathrm{HCO}_{3}$-Austauscher (SLC4A1) exprimiert, die $\mathrm{Cl}^{-}$-Ionen in das Zilienlumen transportieren. Ein weiteres sehr wichtiges ziliäres Protein ist der $\mathrm{Na}^{+} / \mathrm{Ca}^{2+}$ Austauscher $(\mathrm{NaCaX})$, der $\mathrm{Ca}^{2+}$-Ionen aus dem Zilienlumen transportiert (Abbildung 2).

Auch die OR-Rezeptoren des Septalorgans und die TAAR-Rezeptoren der Hauptriechschleimhaut sowie interessanterweise die fünf humanen V1R-Rezeptoren, die der in Primaten auftretenden Pseudogenisierung dieser Familie durch Verlagerung der Expression in die Hauptriechschleimhaut entkommen zu sein scheinen, besitzen einen cAMP-vermittelten Transduktionsweg.

\section{Andere Signaltransduktionswege in olfaktorischen Rezeptorneuronen der Säuger}

Neben der cAMP-vermittelten Transduktionskaskade gibt es in der Hauptriechschleimhaut von Säugetieren auch kleinere Subpopulationen von Rezeptorneuronen, die alternative Transduktionsmechanismen besitzen, unter anderem eine Phospholipase C (PLC), TRP-Kanäle ('transient receptor potential') des Typs C6 und M5, und eine Rezeptor-Guanylylcyclase (GC-D), zusammen mit einem cGMP-abhängigen CNG-Kanal.

In beiden mikrovillären Neuronenpopulationen des Vomeronasalorgans von Säugetieren läuft der Signalweg über eine Phospholipase $\mathrm{C}$, nicht über eine Adenylatcyklase wie in den ziliierten Neuronen der Hauptriechschleimhaut. Apikale Neuronen exprimieren V1RRezeptoren und das G-Protein $\mathrm{G}_{\mathrm{i}}$, während die basalen Neuronen V2R-Rezeptoren und das G-Protein $G$ exprimieren. Die Aktivierung der Phospholipase C führt zur Entstehung von Inositoltrisphosphat ( $\left.\mathrm{IP}_{3}\right)$, Diacylglycerol (DAG) und Arachidonsäure, gefolgt von einer Aktivierung von TRPC2Kanälen. Eine sehr kleine Subpopulation von sensorischen Neuronen des VNO exprimieren Rezeptoren der OR-Familie, aber auch diese Zellen exprimieren das inhibitorische G-Protein $\mathrm{G}_{\mathrm{i}}$ und TRPC2-Kanäle, nicht die für OR-Rezeptoren typischen $\mathrm{G}_{\text {olf }}$ und ACIII. Gleichermaßen läuft die Signaltransduktion der im Grüneberg-Ganglion exprimierten
TAAR-Rezeptoren wie die der dort ebenfalls exprimierten V2R-Rezeptoren über $\mathrm{G}_{\mathrm{i}}$ und $G_{o}$, nicht über $G_{\text {olf }}$ wie in der Hauptriechschleimhaut. Es scheint also so zu sein, dass die Art der Signaltransduktion in erster Linie durch die Rezeptorneuronen bestimmt wird, und dass manche Rezeptorfamilien an verschiedene Kaskaden ankoppeln können.

\section{Olfaktorische Signaltransduktion in niederen Vertebraten}

In Fischen und Amphibien sind die Haupttransduktionswege der Säuger wiederzufinden. So konnte das in der cAMP-vermittelten Transduktionskaskade involvierte G-Protein $\mathrm{G}_{\text {olf }}$ und der cAMP-gesteuerte CNG Kanal in zilientragenden Rezeptorneuronen des Zebrabärblings nachgewiesen werden, während mikrovilläre Rezeptorneuronen $\mathrm{G}_{\mathrm{o}}$ sowie den Kanal TRPC2 exprimieren. Es scheint bei den G-Proteinen allerdings diverse Speziesunterschiede zu geben. So sind auch für Kryptneuronen, den dritten Rezeptorneuronentyp, je nach Fischspezies andere G-Proteine berichtet worden, $G_{i}, G_{o}$ und $G_{q}$, jedoch nie $\mathrm{G}_{\text {olf }}$ Diese Erkenntnisse zeigen, dass nicht cAMP-vermittelte Transduktionswege in Fischen weit verbreitet sind. Auch im Krallenfrosch Xenopus laevis existiert neben Rezeptorneuronen mit dem klassischen cAMP-vermittelten Transduktionsweg eine zweite Population von Neuronen, die Geruchsstoffe cAMP-unabhängig transduzieren.

\section{Adaptation der Signaltransduktion}

Um eine schnelle Reaktion des Organismus auf veränderliche Reize zu ermöglichen, ist die zügige Abschaltung der Signaltransduktion essenziell. In der Tat lässt sich beobachten, dass die Geruchsstoffantwort der Zelle bereits nach wenigen Sekunden nachlässt, wenn Rezeptorneuronen kontinuierlich einem Geruchsstoff ausgesetzt werden. Die Rezeptorneuronen adaptieren und der depolarisierende Rezeptorstrom wird durch mehrere inhibierende Prozesse terminiert: Zum einen binden die in die Zelle eingeströmten $\mathrm{Ca}^{2+}$ Ionen an Calmodulin und die so entstandenen $\mathrm{Ca}^{2+}$-Calmodulinkomplexe binden daraufhin an die CNG-Kanäle, was zu einer verminderten Empfindlichkeit für cAMP führt, und in Folge das Schließen der Kanäle bewirkt, also eine Unterbrechung der Geruchsstoffantwort. Parallel dazu trägt die Aktivierung einer $\mathrm{Ca}^{2+}$ Calmodulin-abhängigen Phosphodiesterase durch die Hydrolyse von cAMP ebenfalls zur Beendigung der Geruchsstoffantwort bei. Zusätzlich wird eine direkte Hemmung der ACIII durch Phosphorylierung mittels einer $\mathrm{Ca}^{2+}$-Calmodulin-abhängigen Kinase diskutiert. Auch die olfaktorischen Rezeptoren selbst werden durch Rezeptor-Kinasen phosphoryliert und dadurch abgeschaltet. In diesem Zusammenhang spielen Arrestine, zytosolische Proteine, die den Rezeptor binden und dadurch sterisch vom G-Protein entkoppeln, eine wichtige Rolle. Diese Proteine sind auch maßgeblich an der Internalisierung von olfaktorischen Rezeptoren beteiligt, was dazu führt, dass die Anzahl der Rezeptoren auf der Zellmembran abnimmt und somit die reizinduzierte Antwort der Rezeptorneuronen gedrosselt wird.

Die Vielzahl von Mechanismen, die für eine rasche Beendigung eines Transduktionsvorgangs vorgesehen sind, suggerieren, dass Rezeptorneurone nicht für eine lang anhaltende Aktivierung, sondern eher für repetitive sehr kurze Stimuli ausgelegt sind.

\section{Elektrische Eigenschaften von olfaktorischen Rezeptorneuronen}

Die durch Aktivierung olfaktorischer Rezeptoren ausgelöste Signaltransduktionskaskade erzeugt ein depolarisierendes Rezeptorpotenzial, das oberhalb eines bestimmten Schwellenpotenzials eine Salve von Aktionspotenzialen auslöst, welche über die Axone der Rezeptorneuronen in den Bulbus olfactorius geleitet werden. Die elektrische Kapazität von Rezeptorneuronen liegt im Bereich von 0.7-35 pF und ihr elektrischer Ruhemembranwiderstand beträgt 1-40 G $\Omega$. Ihr Ruhemembranpotenzial liegt zwischen -85 und $-70 \mathrm{mV}$ und die Membranzeitkonstante liegt zwischen ca. 40 und mehr als 100 ms. Mit diesen elektrischen Eigenschaften genügt theoretisch die Bindung von nur sehr wenigen Geruchsstoffmolekülen, um ein Rezeptorneuron zu aktivieren. Allerdings ist die Lebensdauer von Rezeptor-Geruchsstoffmolekül-Komplexen nur sehr gering $(<$ $1 \mathrm{~ms}$ ) aufgrund der geringen Affinität, die viele Geruchsstoffe zu ihrem Rezeptor haben, und daher ist die Wahrscheinlichkeit, dass die Bindung eines Geruchsstoffmoleküls an einen Rezeptor die Transduktionskaskade aktiviert, trotzdem sehr klein. Man muss allerdings bedenken, dass bei Geruchsstoffkonzentrationen im mikromolaren Bereich, das sind normale physiologische Konzentrationen für olfaktorische Rezeptoren der OR-Familie (Saito et al. 2009), mehrere Millionen Geruchsstoffmoleküle pro Sekunde an ein Zilium, das viele olfaktorische Rezeptoren besitzt, gelangen und dadurch die Wahrscheinlichkeit von wenigstens einigen positiven Transduktionsvorgängen wiederum relativ hoch ist. Man nimmt an, dass ca. $20-35$ positive RezeptorGeruchsstoffmolekül-Bindevorgänge ausreichen, um ein Rezeptorneuron zu aktivieren. 
Interessanterweise weist die gesamte Kaskade einen sehr geringen Verstärkungsfaktor auf, für einen aktivierten Rezeptor wird nur ungefähr ein Golf/ACIII Komplex gebildet.

Bei sensorischen Zellen des VNO, die schon auf Pheromonkonzentrationen ab 0.1 pM reagieren, muss das natürlich anders sein. Bei solch niederen Konzentrationen gelangen im Mittel nur einige wenige Pheromonmoleküle pro Sekunde an Rezeptoren. Die Affinität von Pheromonen zu ihren Rezeptoren muss deshalb sehr hoch sein, um eine lange Lebensdauer der Rezeptor-PheromonmolekülKomplexe zu gewährleisten. Nur so kann es gelingen, genügend positive Transduktionsvorgänge zu erreichen, um die vomeronasalen Rezeptorneuronen zu aktivieren.

Olfaktorische Rezeptorneurone haben in der Regel eine gewisse basale Aktivität, d.h., sie weisen auch in Ruhephasen, wenn keine Geruchsstoffbindung stattfindet, eine bestimmte Aktionspotenzialfrequenz auf. Diese basale Aktivität könnte ihren Ursprung in einer hohen basalen G-Protein- oder ACIIIAktivität haben. Eine gerade erschienene Studie hat gezeigt, dass wohl die olfaktorischen Rezeptoren selbst der Ausgangspunkt der Spontanaktivität von Rezeptorneuronen sein könnten und dass unterschiedliche olfaktorische Rezeptoren verschieden starke Spontanaktivitäten auslösen können. Olfaktorische Rezeptoren könnten demnach nicht nur die Geruchsstoffselektivität eines Rezeptorneurons bestimmen, sondern diesem auch eine einzigartige Identität bezüglich seiner Basalaktivität aufprägen. Diese Ruheaktivität eröffnet den Rezeptorneuronen die Möglichkeit, auch inhibitorisch, d.h. mit einer Hyperpolarisation und einer daraus resultierenden Abnahme ihrer basalen Aktionspotenzialfrequenz auf Geruchsstoffe zu reagieren. Inhibitorische Antworten von Rezeptorneuronen wurden in mehreren Spezies beschrieben. Unter anderem wurden eine geruchsstoffinduzierte Blockierung von CNG-Kanälen und Aktivierung von $\mathrm{Ca}^{2+}$-aktivierten $\mathrm{K}^{+}$-Kanälen als mögliche Mechanismen identifiziert, die inhibitorische Antworten vermitteln.

\section{Periphere Modulierung olfaktorischer Signale}

Die Riechschleimhaut wurde lange Zeit einfach als ein Ort angesehen, an dem einzelne Rezeptorneurone unbeeinflusst von anderen Zellen Geruchsstoffe detektieren und die so gewonnenen Informationen unverarbeitet an den Bulbus olfactorius weiterleiten. Diese allzu simple Sichtweise wurde in den vergangenen Jahren zunehmend widerlegt. Neben den olfaktorischen Rezeptoren wurden in Rezeptorneuronen verschiedener Spezies über 30 weitere G-Protein-gekoppelte und ionotrope Rezeptoren entdeckt, unter anderem purinerge Rezeptoren, adrenerge Rezeptoren, cholinerge Rezeptoren und CannabinoidRezeptoren. Eine Modulierung der Geruchsstoffantworten schon in der Riechschleimhaut konnte unter anderem für Acetylcholin, Adrenalin, Noradrenalin, Cannabinoide und ATP gezeigt werden (Hall 2011). Die Riechschleimhaut kann nicht mehr ausschließlich als Sitz isolierter „Geruchsstoffsensoren“ gesehen werden, sondern muss als komplexes Neuroepithel, dessen Zelltypen sich gegenseitig beeinflussen, verstanden werden.

Die Geruchsstoffsensitivität von olfaktorischen Rezeptorneuronen der Maus wird durch die Aktivierung purinerger Rezeptoren (P2X- und P2Y-Rezeptoren) durch ATP verringert, während eine Hemmung der purinergen Rezeptoren die Geruchsstoffantworten verstärkt (Abbildung 3). Es wurde vermutet, dass eine verletzungsbedingte Freisetzung von ATP die allgemeine Aktivität in der Riechschleimhaut herabsetzen und so zu einer schnelleren Erholung des verletzten Gewebes beitragen könnte. Dazu passt, dass im larvalen Xenopus laevis die Hemmung purinerger Rezeptoren die Proliferationsrate von neuronalen Stammzellen in der Riechschleimhaut reduziert, also ATP die Proliferation und damit die Regeneration nach Verletzung fördern könnte (Abbildung 3).

Cannabinoide verstärken und CannabinoidAntagonisten unterdrücken die Geruchsstoffantworten von olfaktorischen Rezeptorneuronen des Xenopus, vermutlich über CB-1-Cannabinoid-Rezeptoren an Dendriten von Rezeptorneuronen (Abbildung 3). 2-Arachidonylglycerol wurde als ein verantwortliches Endocannabinoid identifiziert, das die Geruchsstoffdetektionsschwelle von Rezeptorneuronen herabsetzt und hauptsächlich von Stützzellen produziert wird, in Abhängigkeit vom Hungerzustand der Tiere. Demnach könnten hungrige Tiere schon geringere Geruchsstoffkonzentrationen wahrnehmen, was die Lokalisierung von Futter erleichtern könnte.

Die Riechschleimhaut vieler Spezies wird autonom innerviert, sowohl von sympathischen als auch parasympathischen Nervenendigungen, die Noradrenalin bzw. Acetylcholin freisetzen. Diese Stoffe beeinflussen zum einen die Stützzellen und Blutgefäße der Riechschleimhaut, modulieren aber auch die Aktivität von Rezeptorneuronen (Abbildung 3). Die Stimulierung sowohl adrenerger als auch cholinerger Rezeptoren auf den Rezeptorneuronen erhöhte die geruchsstoffinduzierten ElektroolfaktogrammSignale. Cholinerge Rezeptoren wurden auf Zilien von Rezeptorneuronen nachgewiesen, wo sie mit olfaktorischen Rezeptoren Komplexe bilden können (Hall 2011).

\section{Konvergenz olfaktorischer Rezeptor- neurone im Bulbus olfactorius}

Ziliierte Rezeptorneurone, die denselben OR exprimieren, konvergieren in einer gemeinsamen Zielregion im Bulbus olfactorius, einem sogenannten Glomerulus, in dem die Synapsen auf die nachgeschalteten Projektionsneuronen, die Mitralzellen liegen (Abbildung 4). Eine rezeptorspezifische genetische Markierung in sogenannten Knock-in-Experimenten hat gezeigt, dass Glomeruli sehr homogen sind bezüglich der innervierenden Rezeptorneuronen, mit Ausnahme der sogenannten Halskettenglomeruli, die ein olfaktorisches Subsystem im posterioren Bulbus olfactorius darstellen. Die Position solcher Glomeruli ist mit einer Genauigkeit von 1-2 Glomerulidurchmessern interindividuell stereotyp, sowohl in Säugern als auch in Fischen, in denen viele Glomeruli schon aufgrund morphologischer Kriterien identifiziert werden können, was in Säugern nur eingeschränkt möglich ist. Damit ergibt sich eine rezeptotope Karte, also eine Abbildung der Rezeptoren auf die Bulbusoberfläche. Interessanterweise hat in Nagern eine spiegelbildliche Verdoppelung dieser Karte stattgefunden, sodass pro olfaktorischem Bulbus jedem Geruchsrezeptor zwei Glomeruli zugeordnet sind (manchmal ist die Konvergenz nicht perfekt und die Zahl der Glomeruli ist etwas höher). Man würde daher über 2000 Glomeruli im Bulbus olfactorius erwarten, was ungefähr mit der anatomisch bestimmten Gesamtzahl zusammenpasst.

Mikrovilläre Neurone zeigen eine reduzierte Konvergenz, die zu einer Handvoll von Mikroglomeruli als gemeinsamem Zielort einer Population von vomeronasalen Neuronen führt. In Säugern sind die Zielgebiete der mikrovillären Neuronen im akzessorischen Bulbus olfactorius zu finden und damit zwar angrenzend, aber klar getrennt von denen der ziliierten Rezeptorneuronen im Hauptbulbus, während bei Fischen die beiden Zielgebiete zwar auch räumlich getrennt, aber doch mehrfach ineinander verschränkt sind. Axone von zilientragenden Rezeptorneuronen, die olfaktorische Rezeptoren der OR-Familie exprimieren, projizieren hauptsächlich in den dorsalen und medialen Teil des Bulbus olfactorius. Mikrovilläre Rezeptorneuronen, die olfaktorische Rezeptoren der V2R-Familie exprimieren, projizieren hauptsächlich in den lateralen Teil des Bulbus olfactorius. In Amphibien projizieren die mikrovillären Neuronen des vomeronasalen Organs wie bei Säugern in den akzessorischen Bulbus olfac- 


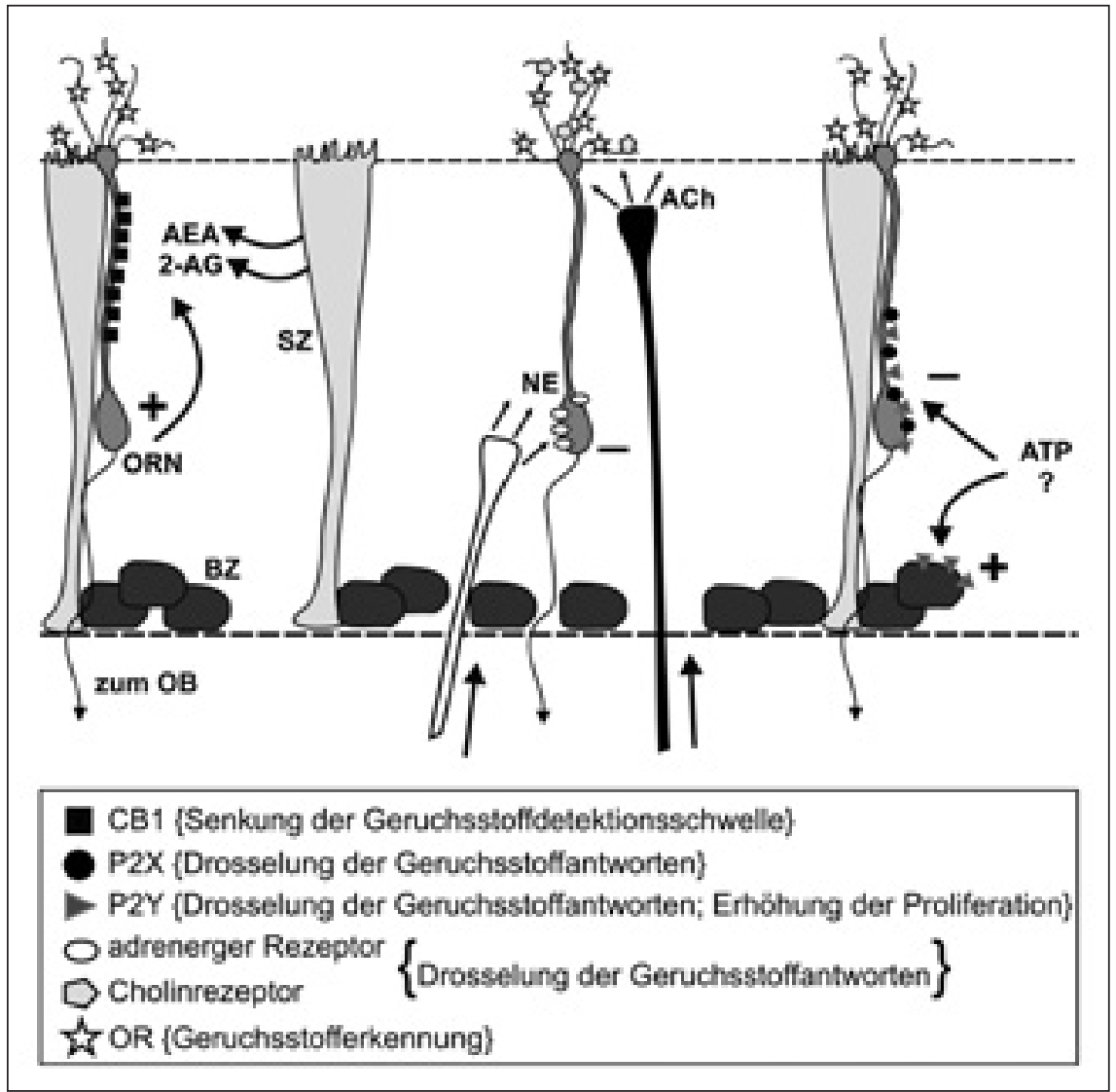

Abb. 3: Periphere Modulierung der Geruchsstoffantworten Schematische Darstellung der Riechschleimhaut und eine Auswahl von Rezeptoren und Substanzen, die olfaktorische Information in der Riechschleimhaut modulieren. Eine genaue Beschreibung kann in der Abbildung selbst und im Text gefunden werden. Verwendete Abkürzungen: 2-AG, 2-Arachidonylglycerol; Ach, Acetylcholin; AEA, Arachidonylethalonamid; ATP, Adenosintriphosphat; BZ, Basalzellen; NE, Noradrenalin; OB, Bulbus olfactorius; ORN, olfaktorisches Rezeptorneuron; SZ, Stützzelle.

torius, aber die mikrovillären Rezeptorneurone der Hauptriechschleimhaut projizieren zusammen mit den ziliierten Neuronen in den Hauptbulbus, wenngleich in unterschiedliche Regionen.

Die weiteren olfaktorischen Organe der Säuger haben ebenfalls spezialisierte Zielgebiete, die ziliierten Neurone des Grüneberg-Ganglions bilden einen Teil der Halskettenglomeruli aus, während andere Halskettenglomeruli von den GC-D exprimierenden Neuronen der Hauptriechschleimhaut angesteuert werden. Die ziliierten Neurone des Septalorgans projizieren in eine kleine Zielregion im ventralen Bulbus olfactorius.

\section{Die Ontogenese der rezeptotopen Karte im Bulbus olfactorius}

Die rund 1000 unterschiedlichen Rezeptorneuron-Subpopulationen der Maus mit ihren rund 2000 Zielglomeruli stellen eine beträchtliche Herausforderung für die axonale Wegfindung dieser Neuronen dar. Die faszinierende Frage ist nun, welche(s) Signal(e) die Axone einer bestimmten Rezeptorneuron-Subpopulation $\mathrm{zu}$ der richtigen Position führen und konvergieren lassen. In der Maus konnte gezeigt werden, dass die olfaktorischen Rezeptoren, die auch auf den Axonterminalen zu finden sind, selbst eine wichtige Rolle bei der Bestimmung des Zielortes der Axone im Bulbus olfactorius spielen. Möglicherweise sind jedoch die olfaktorischen Rezeptormoleküle nicht direkt an der Bestimmung des Zielortes im Bulbus olfactorius beteiligt, sondern lenken durch rezeptorabhängige cAMP-Signale die axonale Projektion. Dabei wird angenommen, dass jeder Rezeptortyp einzigartige Pegel von cAMP-Signalen generiert, die die Transkription von Axon-Lenkungsmolekülen regulieren (Abbildung 4) und dadurch die Zielposition auf der anterior-posterioren Achse im Bulbus olfactorius bestimmen.

Eine weitere Gruppe von Lenkungsmolekülen scheint die Zielposition der Axonter- minalen auf der dorso-ventralen Achse des Bulbus festzulegen. Diese Achse korreliert mit einer dorso-ventralen Anordnung der Rezeptorgenexpression in der Hauptriechschleimhaut. Olfaktorische Rezeptorneurone, die im dorsalen Teil der Riechschleimhaut liegen, projizieren ihre Axone in den dorsalen Bulbus olfactorius, Rezeptorneurone die im ventralen Teil der Riechschleimhaut liegen, projizieren in den ventralen Bulbus olfactorius (Abbildung 4; Mori und Sakano 2011). Damit ist das zweidimensionale Problem der Zielfindung auf zwei eindimensionale Problerme reduziert worden, die durch unabhängige Mechanismen gelöst werden können.

Schließlich erfolgt die Koaleszenz der Axonterminalen in scharf begrenzte Glomeruli, also die Feinabstimmung des Zielgebietes, in aktivitätsabhängiger Weise, wie es für viele Projektionen im Gehirn bekannt ist.

In niederen Vertebraten wie Fischen oder Amphibien sind axonale Lenkungsmechanismen in Rezeptorneuronen weniger gut erforscht, jedoch scheinen ähnliche Lenkungsmoleküle wie in der Maus auch im Zebrabärbling eine wichtige Rolle zu spielen.

\section{Imaging von Geruchsstoffantworten im Bulbus olfactorius}

Durch die Vermessung der Geruchsstoffantworten im Bulbus olfactorius können simultan Aussagen über die Aktivierung einer Vielzahl von Glomeruli, und damit tentativ auch der zugrunde liegenden Rezeptoren, gemacht werden. Sowohl geruchsstoffinduzierte Aufnahme von 2-Deoxyglukose, Kalziummessungen mit eingebrachten fluoreszenten Kalziumindikatoren, Messungen des Membranpotenzials mittels spannungsabhängiger Fluoreszenzfarbstoffe, als auch 'intrinsic imaging', welches geruchsstoffinduzierte Änderungen in den optischen Eigenschaften ungefärbter, nativer Bulbi quantifizieren kann, sind verwendet worden (Johnson und Leon 2007). Obwohl das intrinsic imaging Signal im Wesentlichen indirekte Effekte, wie die lokale Änderung des Blutstroms in der Nähe aktivierter Neuronen misst, ähneln die hiermit erhaltenen Ergebnisse denen der Kalziummessungen, die spezifisch in den präsynaptischen Axonterminalen der Rezeptorneuronen durchgeführt wurden.

Wie bereits für die Rezeptormoleküle und die Rezeptorneuronen beschrieben, konnte auch auf Ebene der Glomeruli im Bulbus olfactorius eine kombinatorische Repräsentation gezeigt werden. Einzelne Geruchsstoffe aktivieren in der Regel mehrere Glomeruli, und einzelne Glomeruli werden meist von mehreren verschiedenen Geruchsstoffen aktiviert. Die Erkennung eines Geruchsstoffs 
kann daher über das komplexe Aktivitätsmuster vieler Glomeruli erfolgen. Zusätzlich zum komplexen Aktivitätsmuster spielt auch der zeitliche Verlauf der Aktivität von Glomeruli bei der Geruchsstofferkennung eine Rolle. Durch diese kombinierte räumliche und zeitliche Kodierung kann das olfaktorische System wesentlich mehr Geruchsstoffe unterscheiden als es olfaktorische Rezeptoren hat, da die so möglichen unterschiedlichen Aktivitätsmuster praktisch unbegrenzt sind. Die relativ geringe Spezifität der olfaktorischen Rezeptoren steigert also die Kodierungskapazität des olfaktorischen Systems um ein Vielfaches.

In einzelnen Fällen sind hochspezifische und hochsensitive Glomeruli beschrieben worden, hierbei handelt es sich meist um Pheromonantworten. Hier ist die Optimierung der Kodierungskapazität vermutlich zugunsten einer Minimierung der Reizschwelle und/oder eines unmittelbareren Auslesens der kodierten Information in den Hintergrund getreten.

Geruchsstoffantworten im Bulbus olfactorius weisen in mehreren Spezies eine deutliche Chemotopie auf, d.h. chemisch verwandte Geruchsstoffe aktivieren Glomeruli in ähnlichen Subregionen des Bulbus olfactorius (Abbildung 4; s. auch Mori und Sakano 2011). Das liegt vermutlich daran, dass Neurone mit sequenzverwandten Rezeptoren tendenziell auch über ähnliche Informationen zur Positionsbestimmung ihrer Glomeruli verfügen.

\section{Einfluss von Geruchsstoffen auf das Verhalten}

Eine beachtliche Vielfalt von Verhaltensweisen wird durch den Geruchssinn gesteuert, von dem Verfolgen von Beute bzw. dem Entkommen vor Fressfeinden und der Lokalisierung von Futterquellen über Partnersuche und Reproduktion bis hin zu vielgestaltigen innerartlichen Kommunikationen. Je nach Spezies ist die Bedeutung des Geruchssinns unterschiedlich groß, aber es gibt quer durch das Tierreich viele Beispiele für geruchsgesteuertes Verhalten, so auch bei Säugern, Reptilien, Amphibien und Fischen. Pheromone, also innerartliche chemische Botenstoffe, regeln wichtige Verhaltensweisen wie Partnerwahl, Eiablage, Nestbau, Mutter-KindBindung, Abort, Flucht und Aggression und werden vorwiegend im vomeronasalen Organ detektiert (Tirindelli et al. 2009). Manche Pheromone werden jedoch über andere olfaktorische Subsysteme wahrgenommen, z.B. Alarmpheromone über das Grüneberg-Ganglion. Neurone der Hauptriechschleimhaut sind an der geruchsvermittelten Steuerung männlicher Aggression beteiligt (Tirindelli et al. 2009).
Besonders gut erforscht sind Pheromone, die bei Mäusen die Wahl des Geschlechtspartners beeinflussen. Es handelt sich zum einen um MHC-Peptide, die durch ihre Rolle in der Antigenpräsentation im Immunsystem bekannt sind, aber auch durch chemosensorische Zellen des VNOs wahrgenommen werden und zum anderen um ESP-Peptide, die durch die Tränendrüse ausgeschieden werden. In beiden Fällen ist je ein spezifischer Geruchsstoffrezeptor aus der V2R-Familie für diese Peptidliganden identifiziert worden (Haga et al. 2010).

Eine weitere Gruppe von Peptiden mit einer mutmaßlichen Rolle als Botenstoffe sind die Formylpeptide, die als Abbauprodukte bakterieller und mitochondrialer Proteine entstehen, in Körpersekreten ausgeschieden werden, und so Hinweise auf den Krankheitszustand von Artgenossen oder die bakterielle Belastung von Futter geben könnten. Ihre Rezeptoren sind in Mäusen als die fünfte und kleinste olfaktorische Rezeptorgenfamilie identifiziert worden und werden in vomeronasalen Rezeptorneuronen exprimiert (Rivière et al. 2009).

In Fischen sind Steroide und Prostaglandine als Pheromone bekannt, die olfaktorisch wahrgenommen werden und das Reproduktionsverhalten steuern. Es ist zu erwarten, dass in den kommenden Jahren zum einen die molekulare Identifizierung von Pheromonen und ihren Rezeptoren große Fortschritte machen wird, und zum anderen die neuronalen Netzwerke entschlüsselt werden können, die das Signal der aktivierten Rezeptorneuronen verarbeiten und bis zur Generierung spezifischen Verhaltens weiterleiten.

\section{Literaturverzeichnis}

Billig, G.M., Pál, B., Fidzinski, P. und Jentsch, T.J. (2011): $\mathrm{Ca}\left(2^{+}\right)$-activated $\mathrm{Cl}(-)$ currents are dispensable for olfaction. Nat Neurosci. 6: 763-9. Haga, S., Hattori, T., Sato, T., Sato, K., Matsuda, S., Kobayakawa, R., Sakano, H., Yoshihara, Y., Kikusui, T. und Touhara, K. (2010): The male mouse pheromone ESP1 enhances female se-

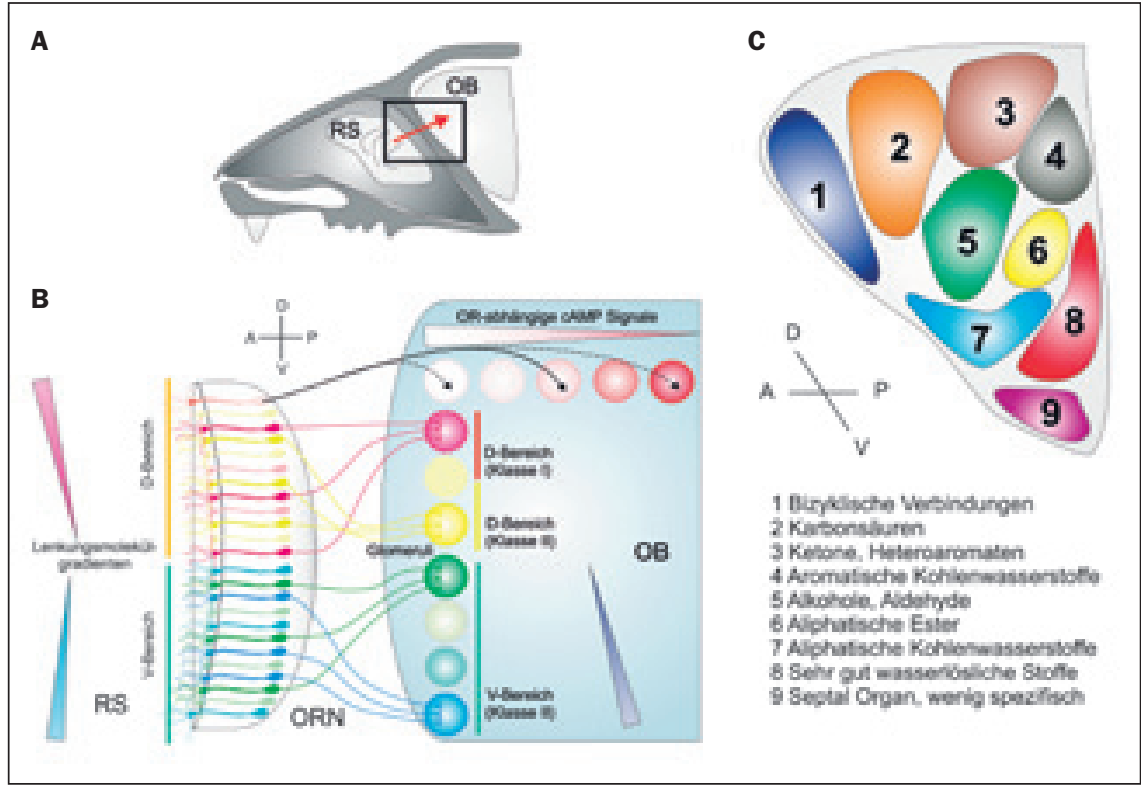

Abb. 4: Entstehung und Eigenschaften der glomerulären Karte im Bulbus olfactorius A) Die schematische Darstellung des Schädels einer Maus zeigt die Lage der Riechschleimhaut (RS) im hinteren Teil der Nasenhöhle. Eine poröse Knochenplatte (Siebbein) bildet die Abtrennung zum Gehirn. Durch die Poren des Siebbeins gelangen die Axone der olfaktorischen Rezeptorneuronen in den Bulbus olfactorius (OB), wo sie in Glomeruli Synapsen bilden. B) Schematische Darstellung, wie die Axone olfaktorischer Rezeptorneuronen (ORN) gleicher Geruchsstoffselektivität (hier gleicher Farbe) geleitet durch verschiedene Signale auf gemeinsame Glomeruli im OB konvergieren. Die Glomeruli sammeln Signale von mehreren tausenden ORN und verschalten sie mit wenigen Mitralzellen (hier nicht gezeigt). Eine genaue Beschreibung dieser schematischen Darstellung kann im Text gefunden werden. C) Schematische Darstellung einer chemotopen olfaktorischen Karte im OB der Ratte (verwendete Daten aus geruchsstoffinduzierter 2-Deoxyglucose-Markierung, s. Johnson und Leon 2007). Verwendete Abkürzungen: A, anterior; V, ventral; P, posterior; D, dorsal. Die Antworten auf verschiedene Substanzgruppen sind über Nummern verschiedenen Positionen im Bulbus zugeordnet. 
xual receptive behaviour through a specific vomeronasal receptor. Nature 466 (7302): 118-22.

Hall, R.A. (2011): Autonomic modulation of olfactory signaling. Sci Signal 4 (155): pe1.

Johnson, B.A. und Leon, M. (2007): Chemotopic odorant coding in a mammalian olfactory system. J Comp Neurol 503 (1): 1-34

Magklara, A., Yen, A., Colquitt, B.M., Clowney, E.J., Allen, W., Markenscoff-Papadimitriou, E., Evans, Z.A., Kheradpour, P., Mountoufaris, G., Carey, C., Barnea, G., Kellis, M. und Lomvardas, S. (2011): An epigenetic signature for monoallelic olfactory receptor expression. Cell 145 (4): 555-70.

Mombaerts, P. (2004): Genes and ligands for odorant, vomeronasal and taste receptors. Nat Rev Neurosci 4: 263-78.

Mori, K. und Sakano, H. (2011): How is the olfactory map formed and interpreted in the Mammalian brain? Аппи Rev Neurosci 34: 467-99.

Nara, K., Saraiva, L.R., Ye, X. und Buck, L.B. (2011): A large-scale analysis of odor coding in the olfactory epithelium. $J$ Neurosci 25 : 9179-91.

Rivière, S., Challet, L., Fluegge, D., Spehr, M.und Rodriguez, I. (2009): Formyl peptide receptorlike proteins are a novel family of vomeronasal chemosensors. Nature 459 (7246): 574-7.

Saito, H., Chi, Q., Zhuang, H., Matsunami, H.und Mainland, J.D. (2009): Odor coding by a Mammalian receptor repertoire. Sci Signal 2 (60): ra9.

Tirindelli, R., Dibattista, M., Pifferi, S. und Menini, A. (2009): From pheromones to behavior. Physiol Rev 89 (3): 921-56.

\section{Danksagung}

Die Arbeiten der Autoren werden gegenwärtig vom DFG Forschungszentrum Molekularphysiologie des Gehirns (CMPB; I.M.), vom Schwerpunktprogramm 1392 (I.M. und S.K) und durch die DFG (KO1046/7, S.I.K.) gefördert.

\section{Kurzbiografien}

Ivan Manzini studierte Biologie an der Universität Modena und Reggio nell' Emilia und promovierte 2003 in der Abteilung von Prof. Dr. Dr. Detlev Schild im physiologischen Institut der Universität Göttingen, in dem er anschließend von 2003 bis 2010 als Post-Doc arbeitete. Seit 2011 leitet er an der Universität Göttingen eine durch das DFG Forschungszentrum Molekularphysiologie des Gehirns (CMPB) geförderte selbstständige Nachwuchsgruppe.

Sigrun Korsching studierte Chemie und Biochemie an der Ludwig-MaximiliansUniversität in München und promovierte 1984 mit einer am Max-Planck-Institut für Psychiatrie (jetzt Neurobiologie), Martinsried, in der Abteilung von Hans Thoenen durchgeführten Arbeit über
Nervenwachstumsfaktoren. Sie ging als Post-Doc ans California Institute of Technology (1986-1988) und nahm danach am Max-Planck-Institut für Entwicklungsbiologie in Tübingen eine Stelle als Nachwuchsgruppenleiter an. In dieser Zeit begann sie, Fragestellungen zum Thema Geruchssinn zu bearbeiten. Seit 1995 ist Sigrun Korsching Professorin am Institut für Genetik der Universität zu Köln.

\section{Korrespondenzadressen}

Ivan Manzini, Ph.D.

Universität Göttingen

DFG Forschungszentrum

Molekularphysiologie des Gehirns (CMPB)

Abteilung Neurophysiologie und

zelluläre Biophysik

Humboldtallee 23

37073 Göttingen

Tel./Fax: +49 551 395913/8399

E-Mail: imanzin@gwdg.de

Prof. Dr. Sigrun Korsching

Universität zu Köln, Institut für Genetik

Zülpicher Straße 47a

50674 Köln

Tel./Fax: +492214704843/5172

E-Mail: sigrun.korsching@uni-koeln.de

\section{"Jugend forscht" - Sonderpreis der Neurowissenschaftlichen Gesell- schaft 2011}

Die Neurowissenschaftliche Gesellschaft vergibt jährlich einen mit $500 €$ dotierten Sonderpreis für ein neurowissenschaft- liches Projekt im Rahmen des Bundeswettbewerbs ,Jugend forscht“. Die Preisträger werden zudem zur Göttinger Tagung

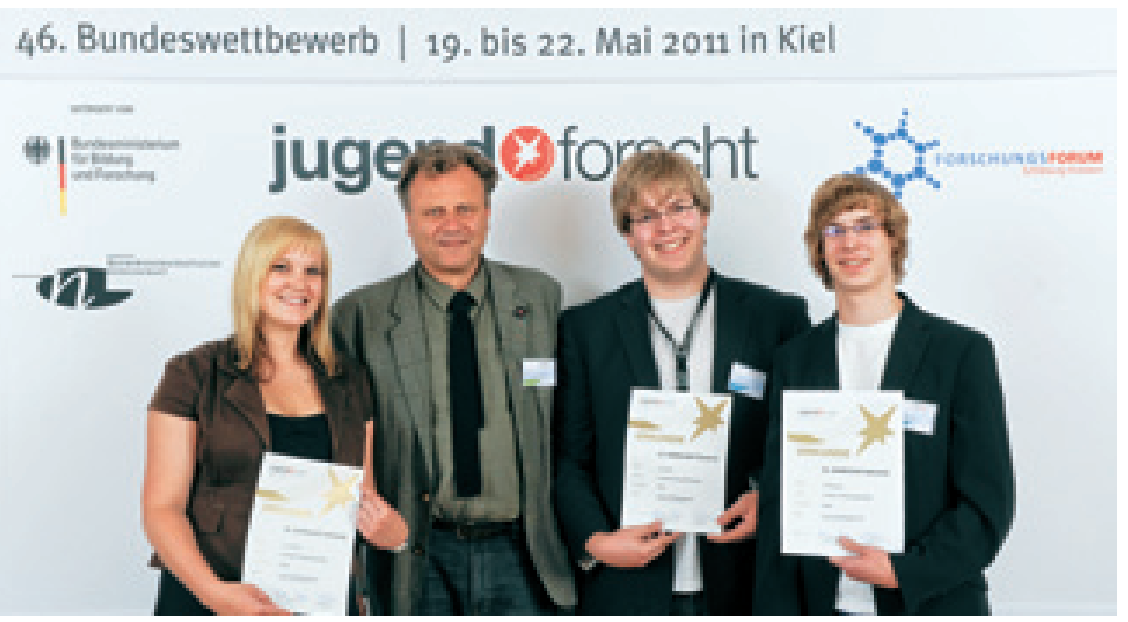

Hans-Joachim Pflüger und die Preisträger: Yvonne Stellmach, Tim Schmäche, Sven Westermann eingeladen und erhalten für ein Jahr ein freies Abonnement für Neuroforum.

Die Preisträger 2011 sind Yvonne Stellmach, Tim Schmäche, beide 18 Jahre alt, und der siebzehnjährige Sven Westermann. Alle drei besuchen das CarlZeiss-Gymansium in Jena/Thüringen. Ihre Arbeit beschäftigt sich mit "Akromegalie und Behandlungsmöglichkeiten".

Akromegalie ist ein krankhafter Überschuss an Wachstumshormonen, der zumeist von einer Geschwulst am Vorderlappen der Hirnanhangdrüse verursacht wird. Die Folge ist ein überwucherndes Wachstum, insbesondere von Bindegewebe und Knochen. Derzeit ist Akromegalie chirurgisch, strahlentherapeutisch oder medikamentös mit Octreotid behandelbar. Gegen dieses Präparat entwickeln Patienten mit fortlaufender Behandlung allerdings oftmals eine Resistenz. Yvonne Stellmach, Tim Schmäche und Sven Westermann haben sich zum Ziel gesetzt, verschiedene Substanzen als Alternative zu Octreotid zu testen. Auf Grundlage ihrer Untersuchungsergebnisse konnten sie konkrete Empfehlungen für das weitere Vorgehen in der Medikamentenforschung geben. 